\title{
Evolution of Evidence-Based Medicine in Stroke
}

\author{
Graeme J. Hankey ${ }^{\mathrm{a}, \mathrm{b}}$ \\ a Medical School, Faculty of Health and Medical Sciences, The University of Western Australia, Perth, WA, Australia; \\ ${ }^{b}$ Department of Neurology, Sir Charles Gairdner Hospital, Perth, WA, Australia
}

\section{Keywords}

Clinical trials $\cdot$ Stroke

\begin{abstract}
The introduction and evolution of evidence-based stroke medicine has realized major advances in our knowledge about stroke, methods of medical research, and patient outcomes that continue to complement traditional individual patient care. It is humbling to recall the state of knowledge and scientific endeavour of our forebears who were unaware of what we know now and yet pursued the highest standards for evaluating and delivering effective stroke care. The science of stroke medicine has evolved from pathophysiological theory to empirical testing. Progress has been steady, despite inevitable disappointments and cul-de-sacs, and has occasionally been punctuated by sensational breakthroughs, such as the advent of reperfusion therapies guided by imaging.
\end{abstract}

(c) 2021 S. Karger AG, Basel

\section{Introduction}

In the early 1980s, I aspired to be a clinical neurologist. My training prioritized clinical expertise, emphasizing proficiency in the diagnosis, management, and care of individual patients. By the mid-1980s, I began to appreciate that caring for individual patients and being an effective clinical neurologist also involved understanding the patient's background, personality, and priorities; their prognosis; and the potential benefits, risks, and costs of treatments that may alter the prognosis [1]. A landmark book by Sackett et al. [2] introduced me to clinical epidemiology, as opposed to "dry" epidemiology, and described how the principles of epidemiology could be applied to individual patients.

\section{Evidence-Based Medicine}

Sackett et al. [3] promoted the practice of "evidencebased medicine" as the judicious application of the best available external evidence, obtained by systematic research, to complement individual clinical expertise in making informed decisions about individual patient care. They [3], and others [4], emphasized that neither evidence nor individual clinical expertise alone were enough and that the best external evidence was determined by the clinical question. The accuracy of a diagnostic test required cross-sectional studies of patients with the disease, the prognosis required observational studies of inception cohorts that were followed up completely, and the effect of treatments (beneficial and harmful) required a systematic review of experiments, ideally randomized controlled trials (RCTs), and if no RCTs available, the next best external evidence [3]. karger@karger.com www.karger.com/ced (c) 2021 S. Karger AG, Base

Karger!
Correspondence to:

Graeme J. Hankey, graeme.hankey@uwa.edu.au 
In the mid-1980s, the principles of epidemiology were also being applied prospectively to populations to study the incidence, causes, and outcome of stroke $[5,6]$ The causes of stroke were numerous, and the outcome of stroke was variable; two-fifths of cases died, one-third remained functionally disabled, and one-quarter were functionally independent at 6 months after stroke $[5,6]$. The outcome was influenced by several prognostic factors, of which treatment was only 1 factor (Table 1) $[5,6]$. There were few, if any, effective treatments for stroke. The implications of these fundamental observations were several.

\section{Matched Controls Were Needed to Distinguish}

Treatment Effects from Natural History

First, the variable natural history of stroke suggested that recovery could occur spontaneously, unrelated to any treatment [6]. Therefore, any assessment of the effect of a treatment would require comparison with an otherwise identical group of untreated controls to distinguish a treatment effect and its size (signal) from the expected prognosis (background noise).

Treatment Effects for Stroke Were Likely to Be Mild or Moderate Overall

Second, because the outcome after stroke was not always fatal or disabling, any effect of treatment on the outcome was not likely to be so dramatic (e.g., signal-tonoise ratio $>10$, as with antibiotics for bacterial septicaemia) that moderate biases could be ruled out as an explanation [7]. The aetiological heterogeneity of stroke also meant there was unlikely to be a single "magic bullet" for stroke, in the same way that there was no single antibiotic for all infections, and no single treatment for all cancers. Hence, an effective treatment given to all stroke patients was likely to have only a mild or moderate overall effect (i.e., less than a 2 -fold difference in the incidence of the outcome between using and not using the treatment) [7].

\section{Moderate Biases Needed to Be Distinguished from}

\section{Moderate Treatment Effects}

Third, the significant effect of several prognostic factors, independent of treatment, on outcome after stroke (Table 1) meant that imbalance in prognostic factors between treated cases and untreated controls could underestimate or nullify a moderate true effect of a treatment (false negative or type 2 error) or erroneously conclude a moderate false effect (false positive or type 1 error). All potential prognostic factors would therefore need to be
Table 1. Prognostic factors that may influence the outcome of patients with stroke

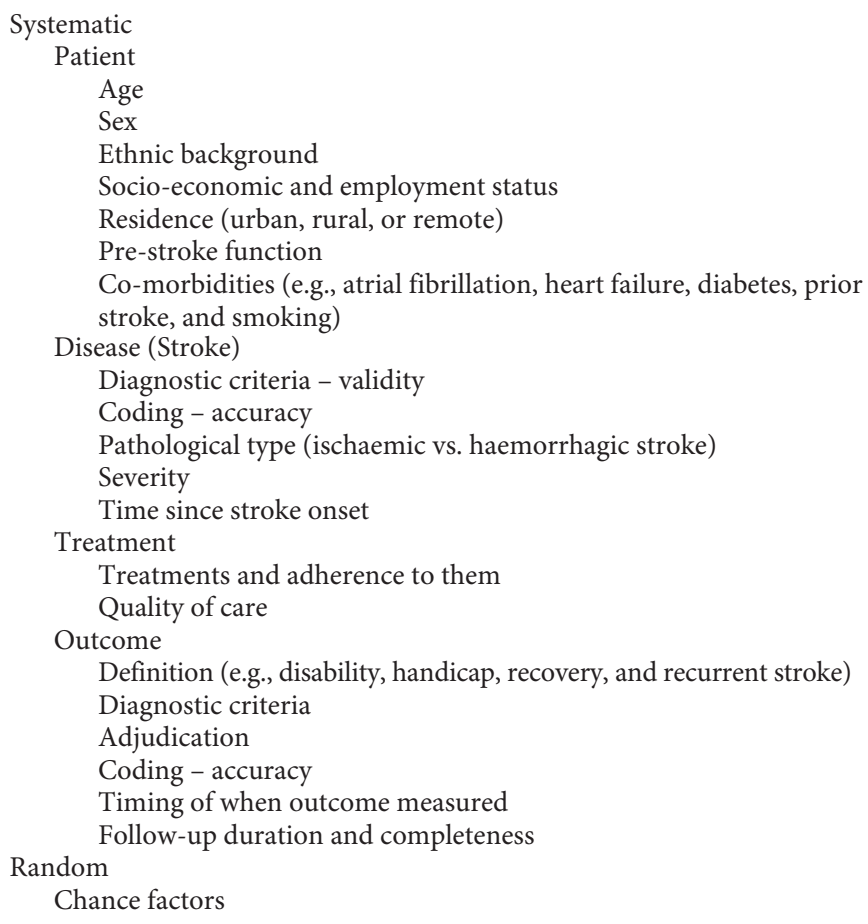

measured, and adjusted if unbalanced, in comparisons of the outcome between cases and controls, before a causal association could be inferred between treatment and outcome. Other potential sources of bias would also need to be minimized, and other aspects of an association between 1 variable (e.g., treatment) and another variable (e.g., outcome) would need to be considered, before deciding whether an association was causal (Table 2) [8]. Hill [8] considered the strongest of these was an experiment, whereby an action (e.g., treatment) reduced or prevented the associated events. However, it took time for methodologically reliable experiments of treatment effects (e.g., RCTs) [9] to become de rigueur in stroke medicine [10].

\section{Methods of Evaluating Treatment Effects}

\section{Observational Epidemiology - The Example of}

Carotid Endarterectomy

Observational cohort and case-control studies [11], driven by pathophysiological concepts, were the mainstay of evaluating interventions to prevent and treat stroke [10]. For example, the safety and effectiveness of 
Table 2. Reasons for an association between 2 variables (e.g., treatment and outcome)

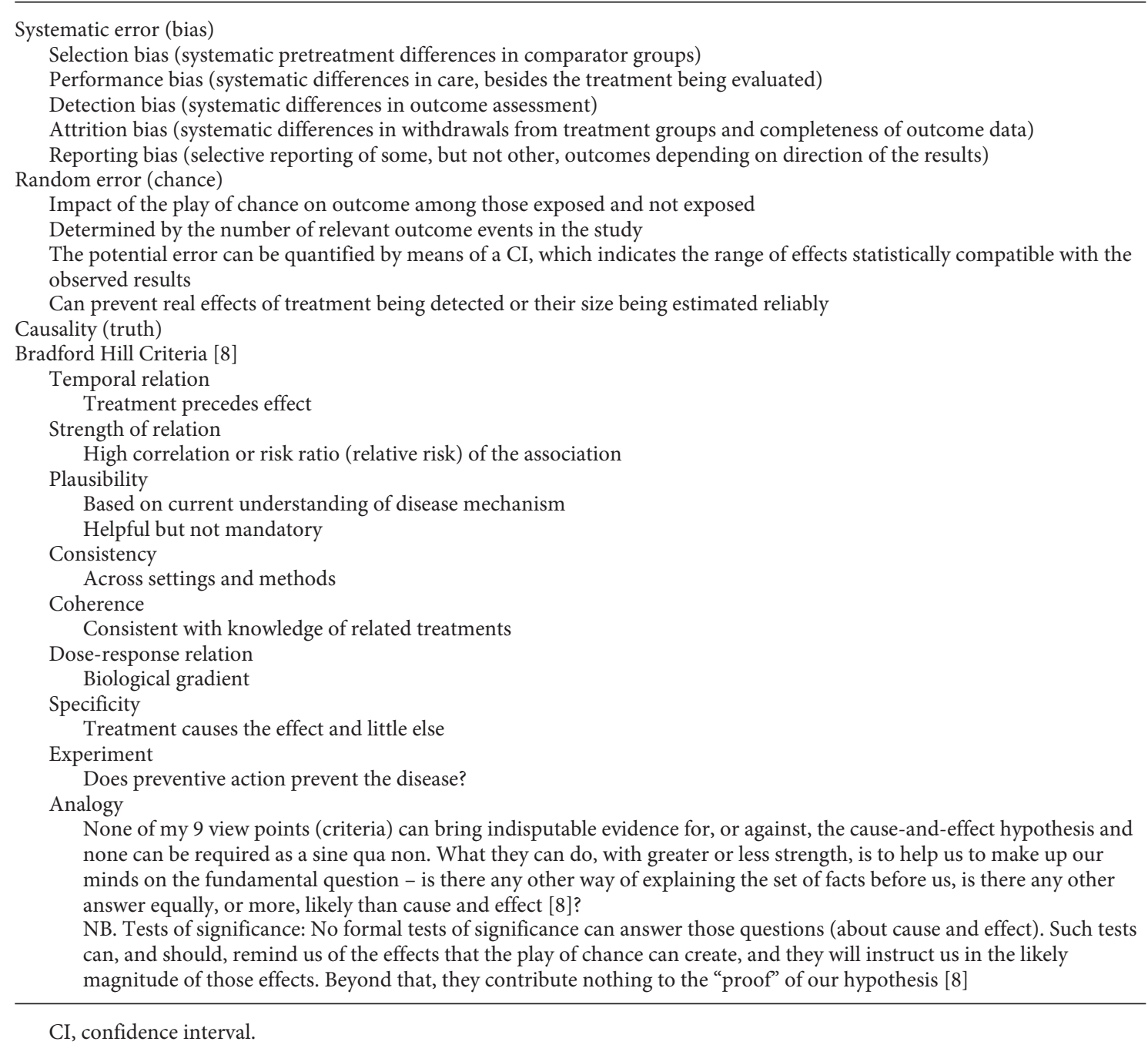

carotid endarterectomy were initially assessed by comparing the outcome of patients who had undergone carotid endarterectomy, usually as part of their hospital care, with the outcome of others who had not (literature, historical, or concurrent controls) [12]. The numerical method was used to compare number of outcome events (strokes) in each group over the average follow-up period, rather than actuarial life tables or the Kaplan-Meier method of survival analysis $[10,12]$. Poor risk patients were less likely to be selected for surgery than good risk patients (indication bias), so patients in surgical series had more favourable outcomes. A range of statistical strategies (e.g., multivariable regression and propensityscore matching) could adjust for imbalance between the surgical and non-surgical groups in measured confound- ing factors but could not remove, with certainty, residual confounding (due to differences between patient groups in unmeasured confounding factors or measured confounding factors that were misclassified or imprecisely measured); confounding by indication (inability to know and adjust for reasons why some patients received the treatment whereas others did not), or reverse causality bias (when the probability of the outcome was causally related to the exposure). Invariably, patients who were treated non-randomly were likely to be systematically different from untreated controls in ways that were not measurable with the data collected, introducing systematic bias into estimates that were wrong, and we could not be sure just how wrong, qualitatively or quantitatively. 
Table 3. Strategies to minimize systematic and random errors

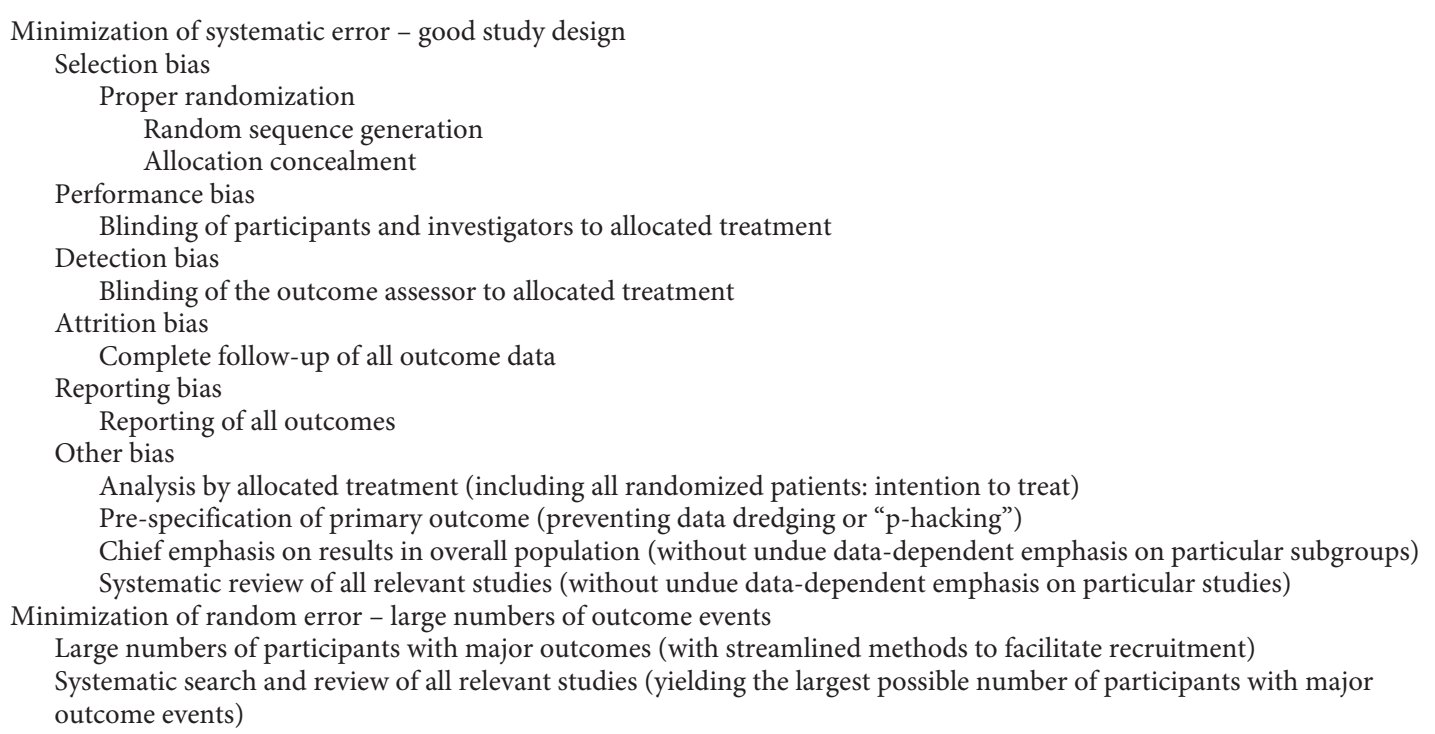

In the same issue of Stroke in 1984, Warlow [12] and Barnett et al. [13] independently called for a good study design to minimize systematic error (bias) and large numbers of primary outcome events to minimize random error (chance) in order to reliably determine the true effect of carotid endarterectomy (Table 3) $[14,15]$. The most efficient method of minimizing systematic error is by concealed randomization (to ensure comparability between treatment groups) and blinded outcome evaluation $[14,15]$. Other fundamentals are good adherence to assigned treatment, complete ascertainment of outcomes, and unbiased statistical analysis.

\section{RCTs - Carotid Endarterectomy, Anticoagulation, Antiplatelet Therapy \\ Carotid Endarterectomy}

The first RCT of carotid endarterectomy, conducted between 1962 and 1968 in 24 US centres under the leadership of Bill Fields [16], was an astonishing effort but the results were inconclusive. The sample size was too small ( $n=316$ patients with TIA and carotid stenosis) to reliably detect or exclude a moderate but clinically worthwhile benefit of surgery; $43 \%$ of the patients had experienced only vertebrobasilar TIA (i.e., asymptomatic carotid stenosis); the results for patients with carotid TIA (i.e., symptomatic carotid stenosis) were not reported; completeness of follow up in each group was not known; the major end point (stroke) was not evaluated "blind" to the allocated treatment; and it was not clear whether the pa-

Evolution of Evidence-Based Stroke Medicine tients were analysed (by intention to treat) in the groups to which they were randomized [16].

Uncertainty about the safety and efficacy of carotid endarterectomy polarized opinions and led to wide variations in surgical policy and practice around the world. In contrast to the UK, carotid endarterectomy became one of the most commonly performed vascular procedures in the USA [12]. Inconsistency between rigorous regulatory requirements for licensing drugs and unregulated uptake of surgical procedures was also exposed [12]. The uncertainty became such a burning issue that it prompted the launch of the European Carotid Surgery Trial (ECST) in 1981 and North American Symptomatic Carotid Endarterectomy Trial (NASCET) in 1987 [17, 18]. Their interim results, published in 1991, resolved uncertainties about the overall balance of benefits and harms of carotid endarterectomy for symptomatic, extracranial carotid stenosis [17, 18]. Moreover, because both trials shared similar methods and friendly collaboration, it was possible to test hypotheses generated from 1 trial in the other independent trial. The individual patient data were pooled to develop and externally validate risk models which enable more precise targeting of carotid revascularisation to patients most likely to benefit, such as those with recent or recurrent symptoms of thromboembolism from an irregular or ulcerated atherosclerotic plaque $[19$, 20].

The ECST and NASCET also illustrated how different trial designs could realize similar and complementary re- 
sults. The ECST had a simple design with few key data requirements recorded on single page forms at baseline and follow-up. Entry was based on the "uncertainty principle," whereby patients were enrolled only if the clinician was substantially uncertain which trial treatment would be most appropriate for their patient $[17,21]$. The uncertainty principle enabled clinicians to provide information and obtain informed consent in a manner similar to routine clinical practice. It facilitated recruitment of a wide range of patients because clinicians had different "grey areas" of uncertainty; for example, some were uncertain about the benefits of carotid endarterectomy in patients with $0-50 \%$ stenosis, others were uncertain about 30 $70 \%$ stenosis, and others were uncertain about $50-99 \%$ stenosis. Hence, the ECST could determine the effectiveness of carotid endarterectomy for all degrees of symptomatic carotid stenosis [17].

\section{Anticoagulation}

In the early 1960s, Hill et al. [22, 23] led 2 RCTs of anticoagulation (phenindione) to prevent recurrent stroke in $>14$-day survivors of presumed ischaemic stroke. The first trial enrolled 142 patients and suggested an adverse effect of anticoagulation in hypertensive patients [22]. The second trial enrolled 131 non-hypertensive patients and reported that anticoagulation increased fatal stroke (possibly haemorrhages) but not non-fatal stroke [23]. Despite several shortcomings of these trials [24], anticoagulation was generally rejected for acute/subacute ischaemic stroke until 35 years later, when a sufficiently large trial showed that early anticoagulation with unfractionated heparin $(5,000$ or $12,500 \mathrm{IU}$ bd) reduced recurrent ischaemic stroke by about 9 (95\% confidence interval [CI]:4-13) per 1,000 patients treated but increased haemorrhagic stroke by about 9 (6-11) per 1,000 patients, with no net reduction in recurrent stroke (odds ratio [OR]: $0.97 ; 0.85-1.11$ ) or death or dependency (OR: $0.99 ; 0.93-$ 1.04) [25].

By contrast, long-term oral anticoagulation for preventing stroke in patients with atrial fibrillation was evaluated systematically by several, well-organized clinical trials in the late 1980s/early 1990s [26-30]. Adjusted-dose warfarin (international normalized ratio [INR]: 2.0-3.0) and antiplatelet therapy were shown to reduce stroke by about two-thirds and one-fifths, respectively, compared to the control [30]. Adjusted-dose warfarin (INR 2.0-3.0) was substantially more efficacious than both antiplatelet therapy alone and the combination of antiplatelet therapy plus low-intensity, fixed-dose warfarin (INR 1.2-1.5) [26-30]. Later, 4 very large RCTs showed non-inferiority of 4 direct oral anticoagulants compared to adjusted-dose warfarin for stroke prevention in atrial fibrillation [31].

Trials of long-term oral anticoagulation for preventing stroke in patients with TIA or minor ischaemic stroke of presumed arterial (non-cardiac) origin were less successful. The first large trial in 1,316 patients was stopped prematurely because high intensity oral anticoagulation (INR: 3.0-4.5) caused an excess of intracranial haemorrhages compared to aspirin $30 \mathrm{mg}$ daily [32]. A follow-up trial found that medium-intensity oral anticoagulation (target INR: 2.0-3.0) reduced ischaemic events (HR: 0.73, 0.52-1.01) but also increased major bleeding (HR 2.56, 1.48-4.43) which offset any benefit on the primary composite outcome (HR: 1.02, 0.77-1.35) compared to aspirin (30-325 mg daily) [33]. A post hoc subgroup analysis of 1,032 patients with non-lacunar, non-cardioembolic, ischaemic stroke in the COMPASS trial now raises the hypothesis that adding low-intensity anticoagulation (rivaroxaban $2.5 \mathrm{mg}$ bd) to aspirin $100 \mathrm{mg}$ daily may be more effective than aspirin alone in preventing recurrent stroke and be acceptablely safe [34].

\section{Antiplatelet Therapy}

The first RCT of antiplatelet therapy (dipyridamole) to prevent stroke was reported in 1969 [35]. Soon after, Bill Fields [36] led the first double-blind RCT of aspirin for secondary stroke prevention between 1972 and 1975 . Among 178 patients with carotid territory TIAs, aspirin significantly reduced the composite of death, cerebral or retinal infarction, or TIA during the first 6 months follow-up but not individual end points. In 1978, the Canadian Cooperative Study reported that among 585 patients with "threatened stroke" (406 men and 179 women), aspirin reduced the risk of stroke or death by $31 \%(p<0.05)$ compared to the placebo [37]. Aspirin was also reported to be effective in men (risk ratio $[R R]=0.52, p<0.005$ ) but not women (RR: $1.42, p=0.35$ ); the subgroup interaction $p$ value was $<0.003$. The conclusion that aspirin was effective for men but not women, based on an underpowered subgroup analysis, became an entrenched belief for more than a decade until the publications of the Antiplatelet Trialists' Collaboration (APT) $[38,39]$.

The January 30,1988, issue of the BMJ contained the first results of the APT [39] and the interim results of the UK-TIA aspirin [40]. The UK-TIA aspirin trial reported that among 2,435 patients with TIA or minor stroke, aspirin reduced the odds of stroke, myocardial infarction (MI), or death compared to the placebo (18\%; 95\% CI: $2-31 \%)$ but not disabling stroke or vascular death $(7 \%$; 95\% CI: $26 \%$ reduction to $18 \%$ increase). The authors la- 
Table 4. Taxonomy of the hierarchy of evidence for treatment efficacy and safety [41]

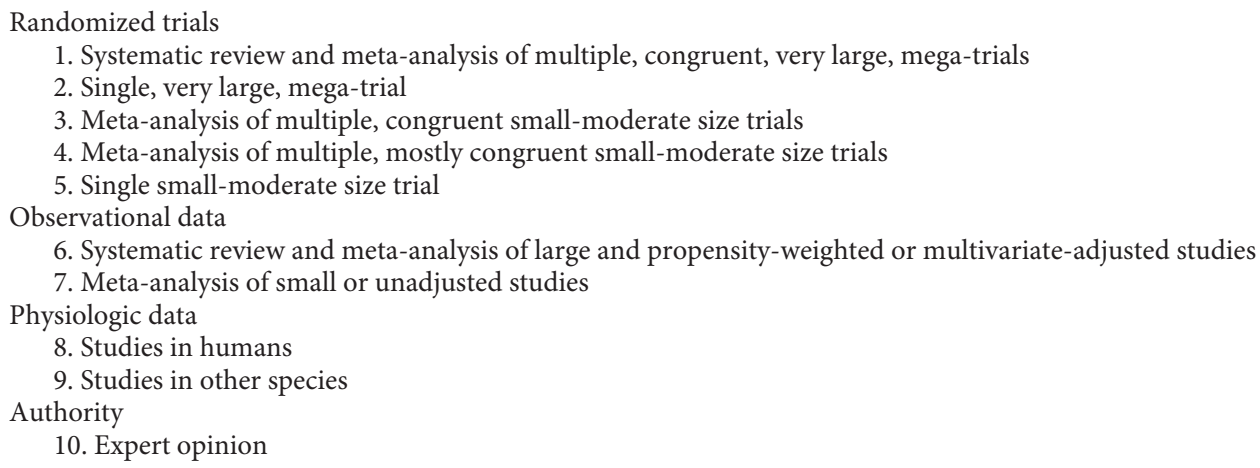

mented, "Having spent nearly 10 years designing, funding, conducting, and analysing this study, we are frustrated to conclude that its results cannot stand alone" [40].

\section{Limitations of Single RCTs}

In addition to suboptimal sample size, other drawbacks of single RCTs were limited generalizability (external validity) and poor reporting [41]. However, although the population of stroke patients studied in any RCT will not be representative of all stroke patients, the proportional effects of the treatment observed in the RCT should be similar, and therefore generalizable, in different stroke populations, unless there is good reason to expect otherwise. If so, the absolute effects of a treatment in local stroke populations can be estimated reliably by applying the proportional effects observed in the RCTs to the absolute risks/incidence of the relevant outcomes recorded in observational studies of local stroke populations [14]. Reporting of RCTs has improved substantially since journals mandated adherence to the Consolidated Standards of Reporting Trials (CONSORT) statements, which provide guidance and checklists for ensuring complete and transparent description of methods and findings $[42,43]$.

\section{Systematic Reviews and Meta-Analyses of RCTs - To} Identify Moderate Treatment Effects

In order to improve precision of the results from single RCTs, without introducing bias, Richard Peto, Tom Chalmers, and Iain Chalmers developed a method of systematically reviewing and collating all related trials in a given field and combining the differences between the treatment group and the control group from each trial into a meta-analysis that produced a summary of the overall effect of interventions from several similar studies $[10,44,45]$. The key assumption was that if a treatment had any definite effect on the incidence or outcome of disease, then the direction of the effect would be similar under different circumstances (i.e., qualitatively similar; positive or negative), whereas the magnitude or size of the effect would be likely to vary (i.e., be quantitatively different) due to clinical and statistical heterogeneity among the trials. The results were graphically represented as "forest plots" with each line depicting the $95 \%$ CI of the RR (or OR) of the outcome for each trial, the square on each line representing the point estimate of the RR (or OR), the size of the square being proportional to the power of the trial, and the overall summary estimate of the RR and its CI at the bottom of the figure [46]. The 95\% CI expressed the range of values that is likely to contain the true effect of the treatment in the trial population, with some degree of uncertainty $[47,48]$. It implied that if the RCTs were to be repeated over and over among random samples from the same population, then $95 \%$ of the calculated intervals would contain the true estimate of the treatment effect. The CI is preferred to $p$ values because estimates of treatment effects/differences derived from controlled comparisons are always subject to some uncertainty (because of both the quality of the data and the play of chance), and the CI captures the degree of statistical uncertainty surrounding the estimate of the treatment effect. Clinicians can then judge whether the likely range of effects would be clinically important or not. In contrast, the $p$ value gives just 2 options - to reject any differences that are not significant or accept those that are, and definitive statements that "no difference exists between treatments" may not be correct [48].

The systematic review and meta-analysis by the APT of 31 RCTs of prolonged antiplatelet therapy in 69,671 
Table 5. Evolution of evidence-base strategies to treat acute stroke and prevent recurrent stroke

\begin{tabular}{|c|c|}
\hline & $\begin{array}{l}\text { Year of initial or } \\
\text { important year }\end{array}$ \\
\hline \multicolumn{2}{|l|}{ Acute treatment } \\
\hline Organized care in stroke units $[52,53]$ & 1993,2012 \\
\hline Graduated compression stockings [55] & 2014 \\
\hline \multicolumn{2}{|l|}{ Ischaemic stroke } \\
\hline Thrombolysis $[60,61]$ & 1995 \\
\hline Aspirin [25] & 1997 \\
\hline Decompressive hemicraniectomy [59] & 2007 \\
\hline Aspirin + clopidogrel $[55,62]$ & 2013 \\
\hline Endovascular thrombectomy $[59,60]$ & 2015 \\
\hline Extending time window and perfusion imaging for EVT [56] & 2018 \\
\hline Extending time window and perfusion imaging for thrombolysis [56] & 2019 \\
\hline Aspirin + ticagrelor [62] & 2020 \\
\hline \multicolumn{2}{|l|}{ Haemorrhagic stroke } \\
\hline Surgery [55] & 2005,2013 \\
\hline Blood pressure-lowering [55] & 2013 \\
\hline \multicolumn{2}{|l|}{ Secondary prevention } \\
\hline \multicolumn{2}{|l|}{ Atherothromboembolism } \\
\hline Aspirin [37-39] & 1978,1988 \\
\hline Carotid endarterectomy $[17,18]$ & 1991 \\
\hline Clopidogrel [72] & 1996 \\
\hline Aspirin + extended release dipyridamole [55] & 1996,2006 \\
\hline Blood pressure-lowering [76] & 2001 \\
\hline Cholesterol-lowering $[77,78]$ & 2002,2006 \\
\hline Cilostazol [55] & 2010 \\
\hline Extracranial carotid stenting [55] & 2011 \\
\hline Pioglitazone [55] & 2016 \\
\hline \multicolumn{2}{|l|}{ Cardiogenic embolism } \\
\hline Anticoagulation with warfarin [29] & 1993 \\
\hline Anticoagulation with direct oral anticoagulants [31] & 2009 \\
\hline Patent foramen ovale closure [61] & 2017 \\
\hline
\end{tabular}

patients showed that antiplatelet therapy reduced the odds of major vascular events by $23 \% \pm 2 \%$ (standard deviation) $(2 p<0.0001)$ compared to control [39]. With an overall treatment effect of $>10$ times the standard deviation $(z$ value $>10)$, there was sufficient statistical power to undertake reliable and meaningful pre-specified subgroup analyses and show more definitively that antiplatelet therapy reduced the odds of major vascular events consistently among men $(24 \pm 2 \%)$ and women $(20 \pm 5 \%)$ [39]. The findings from the APT changed policy and practice, extending antiplatelet therapy to women, as well as men, at high vascular risk [39]. The contrast in conclusions from subgroup analyses in the smaller Canadian Cooperative Study [37] and the larger APT [39] was an early lesson in the potential perils of subgroup analysis, as described in a superb three-part series by Rothwell et al. $[20,49,50]$.

The systematic review and meta-analysis of RCTs has since become the tip of the pyramid (highest tier) of the hierarchy of evidence and grading systems for rating the level (quality) of the evidence and class (strength) of recommendations for interventions (Table 4) [41, 51]. Its methodology was soon embraced by stroke clinicians and revealed the benefits of stroke units $[52,53]$ and decompressive surgery for space-occupying hemispheric infarction [54]. It has subsequently been utilized to more precisely evaluate the effects of other treatments for stroke $[55,56]$, including thrombolysis $[57,58]$, endovascular thrombectomy $[59,60]$, patent foramen ovale closure [61], and dual antiplatelet therapy [62] (Table 5).

Limitations of Systematic Reviews and Meta-Analyses

Limitations of systematic reviews and meta-analyses include potential for publication bias, study quality bias, heterogeneity between studies, reporting bias, poor reporting, and outdated reviews [41, 63-65]. Publication bias arises if studies showing no significant treatment effect ("negative" trials) were less likely to be published, and included in meta-analyses, than "positive" trials, leading to overestimation of the true treatment effect in metaanalyses [63]. Publication bias can be detected sometimes by asymmetrical funnel plots (scatter plots) of effect size against sample size (precision) of the individual studies [66]. Registration of trials (e.g., https://clinicaltrials.gov/) 
now enables reviewers to identify all trials and flag trials where no results were published.

Inclusion of poor quality studies, as assessed by high risk-of-bias judgements (for sequence generation, allocation concealment, blinding, and incomplete data) [64], in systematic reviews has been associated with exaggerated estimates of treatment effects [67], emphasizing the importance of assessing the methodological quality of the trials included in a meta-analysis [64] and conducting a sensitivity analysis of the results according to methodological quality. Systematic reviews may also be biased by selective inclusion and reporting of outcomes and analyses [65]. Selective inclusion occurs when there are multiple estimates of treatment effect in a trial and the choice of estimate is based on the statistical significance, magnitude, or direction of the results. Selective reporting occurs when only a subset of outcomes and analyses are reported, based on the results, rather than reporting all protocol-defined outcomes.

Reporting of systematic reviews and meta-analyses has improved following consensus recommendations in the Preferred Reporting Items for Systematic reviews and Meta-Analyses (PRISMA) statement [68]. The need to regularly update systematic reviews to incorporate the results of recent RCTs led to the Cochrane Collaboration (https://www.cochrane.org/) and Library (https://www. cochranelibrary.com/) $[69,70]$ and a stroke review group within it, which has informed evidence-based stroke medicine for $>25$ years [71].

\section{Mega-Trials - To Identify Moderate Treatment Effects}

Another strategy to improve precision in evaluating treatments with likely moderate, but clinically important, effects has been to conduct very large mega-trials. One of the first mega-trials was the CAPRIE trial which showed that, among 19,185 patients with previous atherosclerotic ischaemic stroke $(n=6,431)$, MI $(n=6,302)$, or peripheral arterial disease $(n=6,432)$, long-term clopidogrel reduced the incidence of ischaemic stroke, MI, or vascular death by $8.7 \%$ (95\% $\mathrm{Cl} 0.3-16.5 \%$; $p=0.043)$ compared to aspirin [72]. The CAPRIE trial also produced another controversy from subgroup analysis. Although only powered to reliably detect (or exclude) the mild observed treatment effect in the whole study cohort, and not each of the 3 clinical subgroups, it was reported that clopidogrel reduced the relative risk of the primary outcome by 7. $3 \%$ (95\% CI: -5.7 to $18.7 \%)$ and $23.8 \%(8.9-36.2 \%)$ in patients with prior stroke and peripheral arterial disease, respectively, but increased the relative risk by $3.7 \%(22.1$ to $-12.0 \%)$ in patients with prior MI [72]. As a test of het-

Evolution of Evidence-Based Stroke Medicine erogeneity of these 3 treatment effects was statistically significant ( $p=0.042)$, it was suggested "that the true benefit (of clopidogrel) may not be identical across the 3 clinical subgroups" [72]. Consequently, clopidogrel was considered by many cardiologists to be no more effective than aspirin for patients with MI and not used by cardiologists until 15 years later, when the Clopidogrel in Unstable angina to prevent Recurrent Events (CURE) trial showed that adding clopidogrel to aspirin in 12,562 patients with acute coronary syndromes reduced the composite of stroke, MI, or cardiovascular death by $20 \%$ (1028\%) [73]. Clopidogrel is now one of the most widely used drugs in cardiology.

The first mega-trials in acute stroke treatment were the International Stroke Trial [25], which enrolled 19,435 patients from 467 hospitals in 36 countries, and the Chinese Aspirin Stroke Trial [74], which enrolled 21,106 patients from 413 Chinese hospitals. They collectively showed that aspirin, started within $48 \mathrm{~h}$ of ischaemic stroke onset, prevented 10 (standard deviation 3 ) deaths or recurrent strokes per 1,000 during the first few weeks $(2 p=0.001)$ $[25,74]$. They lay the foundations (methodology, infrastructure, and networks) for subsequent large trials of stroke treatments [75].

In 2001, the PROGRESS trial reported that, among 6,105 patients with prior stroke or TIA, a perindoprilbased antihypertensive regimen lowered blood pressure by $9 / 4 \mathrm{~mm} \mathrm{Hg}$ and reduced stroke risk by $28 \%$ (95\% CI: 17-38\%) compared to placebo [76]. The proportional reductions in stroke risk were consistent in hypertensive (relative risk reduction: $32 \%$ ) and non-hypertensive (relative risk reduction: $27 \%$ ) participants ( $p$ for homogeneity $=0.7$ ), but the absolute risk reductions were greater in hypertensive participants (5.2\% [11.1\% active vs. $16.2 \%$ placebo]) than in non-hypertensive (2.4\% [ $9.1 \%$ active vs. $11.5 \%$ placebo]) because hypertensive patients are generally at higher absolute risk of stroke than non-hypertensive patients. The following year, the Heart Protection study reported that, among 3,280 participants with prior ischaemic stroke, simvastatin $40 \mathrm{mg}$ daily reduced the risk of major vascular events by $20 \%$ (95\% CI: 8-29\%) compared to placebo. Again, the proportional reduction in risk of one-fifth was consistent, irrespective of baseline blood cholesterol concentration, but the absolute risk reduction was higher among participants with higher blood cholesterol concentrations [77]. The SPARCL trial replicated these results in 4,731 patients with prior stroke or TIA; atorvastatin $80 \mathrm{mg}$ daily reduced the risk of major cardiovascular events by $20 \%$ ( $95 \%$ CI: $8-31 \%$ ) compared to placebo [78]. 
Smaller RCTs - To Identify Large Treatment Effects

Although large RCTs were needed to reliably identify and distinguish moderate treatment effects of medical (drug) therapies for stroke from moderate biases and random errors, smaller RCTs proved sufficient to reliably identify larger treatment effects of surgical and other mechanical interventions for stroke that clearly exceeded any potential moderate errors [17-19, 59-61]. For example, endovascular thrombectomy for acute ischaemic stroke caused by anterior-circulation large-artery occlusion had such a dramatic treatment effect in reducing death and dependency ( $m R S>2$ ) at 90 days ( $47 \%$ control vs. $30 \%$ thrombectomy; RR: 1.56 ; $95 \%$ CI: $1.38-1.75)$ in a total of 1,771 patients enrolled in 7 RCTs that single RCTs with as few as 70 randomized patients had sufficient statistical power to report a statistically significant treatment effect of thrombectomy ( $71 \%$ control vs. $40 \%$ thrombectomy; RR: $1.79 ; 1.13-2.82)$ that was consistent with the other 6 trials [59]. Closure of a patent foramen ovale in patients aged $<60$ years with cryptogenic ischaemic stroke also had a dramatic treatment effect, substantially reducing recurrent ischaemic stroke compared to antiplatelet therapy alone (1.3\% PFO occlusion plus antiplatelet vs. $10 \%$ antiplatelet; OR: 0.12; and 95\% CI: 0.04-0.27) [61]. The astonishing effectiveness of these mechanical interventions, or "plumbing" procedures, is a salutary reminder of potential power of investing in simple clinical innovation and technological advances to further reduce the burden of stroke [79].

\section{Neutral and Negative Trials}

Between the mid-1980s and mid-2000s, many positive results in pre-clinical studies of stroke therapies failed to translate to positive results in large clinical RCTs, and some therapies were even harmful $[80,81]$. Reasons included poor pre-clinical experimental design, conduct, delivery, and clinical oversight. The Stroke Therapy Academic Industry Roundtable (STAIR) responded by publishing standards for preclinical drug development [82] and recommendations for advancing the development of acute stroke therapies [83]. They have facilitated improvements in RCT design and delivery, including preregistration of trials, stratification and minimization at randomization, blinding of treatment and outcomes, and analysis by ordinal logistic regression.

\section{Unpublished Trials}

A substantial body of evidence relevant to stroke practice and research remains unpublished. A systematic review of 940 completed trials of pharmacological interven- tions for acute ischemic stroke (1955-2008) found that 125 trials (19.6\%, 95\% CI: 16.5-22.6\%), involving 16,058 patients, were completed but not published in full [84]. Investigators have a responsibility to ensure that the full methods and results of their RCTs are reported and publically available within a reasonable interval of trial completion.

\section{Current Status and Future Directions}

Now that the rationale and methods for robust clinical trials are accepted, their conduct has been discouraged and compromised by increasing rules, regulation, bureaucracy, governance, complexity, and costs. The intention of research regulation and governance - to protect research participants (their safety, tissues, and data) and improve the reliability of the results - is imperative. Further, it is accepted that regulation and governance is an important way of setting and monitoring quality standards. However, approval systems have been complex, rules and regulations have been interpreted and enforced inconsistently, and assessment of studies has not always been proportionate to the risks of the research to participants [85]. Excessive attention to rules, rather than scientific principles, may neither improve patient safety nor study quality $[14,86]$.

Potential solutions are to simplify, centralize, and harmonize the application process consistently across regulatory bodies and countries, tailor regulatory and governance requirements in proportion to potential harms to the patients, and encourage research centres to network and collaborate in their endeavours to deal professionally and efficiently with regulations and integrate research with clinical practice $[85,87,88]$. The Clinical Trials Transformation Initiative has developed a framework of principles for building quality into the scientific and operational design of clinical trials so that trials are feasible to conduct, participants are safeguarded, important errors are prevented rather than remediated, and results are reliable $[89,90]$. Opportunities to improve the quality and efficiency of RCTs also include ensuring trial guidelines are based on scientific principles, accessing established registries and electronic health-care records to recruit a broad range of patients rapidly, and implementing interactive electronic case report forms and digital technologies (e.g., smartphones and telehealth) to facilitate real-time monitoring and improve protocol adherence, completeness of follow-up, and trial quality [14, 91]. 


\section{Conclusion}

The introduction and evolution of evidence-based stroke medicine has realized major advances in our knowledge about stroke, methods of medical research, and outcomes for patients that continue to complement traditional individual patient care. It is humbling to recall the state of knowledge and scientific endeavour of our predecessors who were unaware of what we know now and yet pursued the highest standards for evaluating and delivering effective stroke care. The science of stroke medicine has evolved from pathophysiological theory to empirical testing. Progress has been steady, despite inevitable disappointments and cul-de-sacs, and has occasionally been punctuated by sensational breakthroughs, such as the advent of reperfusion therapies guided by imaging. My account of the evolution of evidence-based stroke medicine is a personal reflection that is far from comprehensive but is in the spirit of celebrating 30 years since the publication of the first issue of Cerebrovascular Diseases and the honour of contributing to it [92].

\section{Acknowledgments}

I am grateful to Professors Charles Warlow, Peter Sandercock, Jan van Gijn, Geoffrey Rose, Henry Barnett, and David Sackett for teaching me about evidence-based medicine.

Published in Celebration of the 30th Anniversary of the inception of Cerebrovascular Diseases 1991-2021.

\section{Conflict of Interest Statement}

Prof. Hankey trained in neurology and stroke medicine at the Royal Perth Hospital, Mayo Clinic, and University of Edinburgh. He is a senior advisory board member of Cerebrovascular Diseases and a member of the Cochrane Stroke Review Group. He reports personal honoraria outside the submitted work from the American Heart Association (honoraria for serving as the associate editor of Circulation), AC Immune (Chair, Data Safety Monitoring Committee, of ACI-24-701 and AC-35-1201 trials of immune therapies for Alzheimer's disease), Bayer (lecturing at sponsored scientific symposia and consulting on advisory boards about stroke prevention in atrial fibrillation), Bristol Myers Squibb (Executive Steering Committee of the now ceased PARFAIT trial of a PAR4 antagonist for acute TIA and minor ischemic stroke), and Medscape (consulting about physician education regarding thrombosis and antithrombotic therapy).

\section{Funding Sources}

The author received no funding for this study.

\section{References}

1 Caplan LR. The effective clinical neurologist. Boston: Blackwell; 1990.

2 Sackett DL, Haynes RB, Tugwell P. Clinical epidemiology: a basic science for clinical medicine. Boston: Little Brown and Company; 1985. ISBN : 0316765953.

3 Sackett DL, Rosenberg WM, Gray JA, Haynes RB, Richardson WS. Evidence based medicine: what it is and what it isn't. BMJ. 1996; 312:71-2.

4 Caplan LR. Evidence and the effective clinical neurologist: the 2009 H. Houston Merritt lecture. Arch Neurol. 2011;68:1252-6.

5 Bamford J, Sandercock P, Dennis M, Warlow C, Jones L, McPherson K, et al. A prospective study of acute cerebrovascular disease in the community: the Oxfordshire Community Stroke Project 1981-86. 1. Methodology, demography and incident cases of first-ever stroke. J Neurol Neurosurg Psychiatry. 1988; 51:1373-80.

6 Wade DT, Hewer RL. Functional abilities after stroke: measurement, natural history and prognosis. J Neurol Neurosurg Psychiatry. 1987;50:177-82.

7 Glasziou P, Chalmers I, Rawlins M, McCulloch P. When are randomised trials unnecessary? Picking signal from noise. BMJ. 2007; 334:349-51.
8 Hill AB. The environment and disease: association or causation? Proc R Soc Med. 1965; 58:295-300.

9 Collins R, MacMahon S. Reliable assessment of the effects of treatment on mortality and major morbidity, I: clinical trials. Lancet. 2001;357:373-80.

10 Van Gijn J. Chapter 2. Development of knowledge about cerebrovascular disease. In: Hankey GJ, Macleod M, Goreleck PB, Chen C, Caprio FZ, Mattle H, editors. Warlow's stroke: practical management. 4th ed. John Wiley \& Sons, Ltd; 2019. Identifier: LCCN 2018039802 (print).

11 MacMahon S, Collins R. Reliable assessment of the effects of treatment on mortality and major morbidity, II: observational studies. Lancet. 2001;357:455-62.

12 Warlow C. Carotid endarterectomy: does it work? Stroke. 1984;15:1068-76.

13 Barnett HJ, Plum F, Walton JN. Carotid endarterectomy: an expression of concern. Stroke. 1984;15:941-3.

14 Collins R, Bowman L, Landray M, Peto R. The magic of randomization versus the myth of real-world evidence. N Engl J Med. 2020;382: 674-8.

15 Yusuf S, Collins R, Peto R. Why do we need some large, simple randomized trials? Stat Med. 1984;3:409-22.
16 Fields WS, Maslenikov V, Meyer IS, Hass WK, Remington RD, Macdonald M. Joint study of extracranial arterial occlusion. V. Progress report of prognosis following surgery or nonsurgical treatment for transient cerebral ischemic attacks and cervical carotid artery lesions. JAMA. 1970;211:1993-2003.

17 Warlow C; European Carotid Surgery Trialists' Collaborative Group. MRC European Carotid Surgery Trial: interim results for symptomatic patients with severe (70-99\%) or with mild (0-29\%) carotid stenosis. European Carotid Surgery Trialists' Collaborative Group. Lancet. 1991;337:1235-43.

18 North American Symptomatic Carotid Endarterectomy Trial Collaborators, Barnett HJM, Taylor DW, Haynes RB, Sackett DL, Peerless SJ, et al. Beneficial effect of carotid endarterectomy in symptomatic patients with high-grade carotid stenosis. N Engl J Med. 1991;325:445-53.

19 Rothwell PM, Eliasziw M, Gutnikov SA, Warlow CP, Barnett HJ; Carotid Endarterectomy Trialists Collaboration. Endarterectomy for symptomatic carotid stenosis in relation to clinical subgroups and timing of surgery. Lancet. 2004;363:915-24. 
20 Rothwell PM, Mehta Z, Howard SC, Gutnikov SA, Warlow CP. Treating individuals 3: from subgroups to individuals: general principles and the example of carotid endarterectomy. Lancet. 2005;365:256-65.

21 Peto R, Baigent C. Trials: the next 50 years Large scale randomised evidence of moderate benefits. BMJ. 1998;317:1170-1.

22 Hill AB, Marshall J, Shaw DA. A controlled clinical trial of long-term anticoagulant therapy in cerebrovascular disease. Q J Med. 1960; 29:597-609.

23 Hill AB, Marshall J, Shaw DA. Cerebrovascular disease: trial of long-term anticoagulant therapy. Br Med J. 1962;2:1003-6.

24 Jonas S. Anticoagulant therapy in cerebrovascular disease: review and meta-analysis. Stroke. 1988;19:1043-8.

25 International Stroke Trial Collaborative Group. The International Stroke Trial (IST): a randomised trial of aspirin, subcutaneous heparin, both, or neither among 19,435 patients with acute ischaemic stroke. Lancet. 1997;349:1569-81.

26 Stroke Prevention in Atrial Fibrillation Investigators. The Stroke Prevention in Atrial Fibrillation Study: final results. Circulation. 1991;84:527-39.

27 Stroke Prevention in Atrial Fibrillation Investigators. Warfarin versus aspirin for prevention of thromboembolism in atrial fibrillation: Stroke Prevention in Atrial Fibrillation II Study. Lancet. 1994;343:687-91.

28 Stroke Prevention in Atrial Fibrillation Investigators. Adjusted-dose warfarin versus lowintensity, fixed-dose warfarin plus aspirin for high-risk patients with atrial fibrillation: Stroke Prevention in Atrial Fibrillation III randomised clinical trial. Lancet. 1996;348: 633-8.

29 European Atrial Fibrillation Trialists' Study Group. Secondary prevention of vascular events in patients with nonrheumatic atrial fibrillation and recent transient ischemic attacks or minor ischemic stroke. Lancet. 1993 342:1255-62.

30 Hart RG, Pearce LA, Aguilar MI. Meta-analysis: antithrombotic therapy to prevent stroke in patients who have nonvalvular atrial fibrillation. Ann Intern Med. 2007;146:857-67.

31 Ruff CT, Giugliano RP, Braunwald E, Hoffman EB, Deenadayalu N, Ezekowitz MD, et al. Comparison of the efficacy and safety of new oral anticoagulants with warfarin in patients with atrial fibrillation: a meta-analysis of randomised trials. Lancet. 2014;383:95562.

32 Stroke Prevention in Reversible Ischemia Trial (SPIRIT) Investigators. A randomized trial of anticoagulants versus aspirin after cerebral ischemia of presumed arterial origin. The Stroke Prevention in Reversible Ischemia Trial (SPIRIT) Study Group. Ann Neurol. 1997; 42:857-65.

33 ESPRIT Study Group; Halkes PH, van Gijn J, Kappelle LJ, Koudstaal PJ, Algra A. Medium intensity oral anticoagulants versus aspirin after cerebral ischaemia of arterial origin (ESPRIT): a randomised controlled trial. Lancet Neurol. 2007;6:115-24.

34 Sharma M, Hart RG, Connolly SJ, Bosch J, Shestakovska O, Ng KKH, et al. Stroke outcomes in the COMPASS Trial. Circulation. 2019;139:1134-45.

35 Acheson J, Danta G, Hutchinson EC. Controlled trial of dipyridamole in cerebral vascular disease. Br Med J. 1969;1:614-5.

36 Fields WS, Lemak NA, Frankowski RF, Hardy RJ. Controlled trial of aspirin in cerebral ischemia. Stroke. 1977;8:301-14.

37 Canadian Cooperative Study Group. A randomized trial of aspirin and sulfinpyrazone in threatened stroke. N Engl J Med. 1978;299: 53-9.

38 Antiplatelet Trialists' Collaboration. Secondary prevention of vascular disease by prolonged antiplatelet treatment. Antiplatelet Trialists' Collaboration. Br Med J. 1988;296: 320-31.

39 Antiplatelet Trialists' Collaboration. Collaborative overview of randomised trials of antiplatelet therapy--I: Prevention of death, myocardial infarction, and stroke by prolonged antiplatelet therapy in various categories of patients. Antiplatelet Trialists' Collaboration. BMJ. 1994;308:81-106.

40 UK-TIA Study Group. United Kingdom transient ischaemic attack (UK-TIA) aspirin trial: interim results. UK-TIA Study Group. Br Med J. 1988;296:316-20.

41 Saver JL, Hankey GJ. Chapter 2. Understanding evidence. In: Saver JL, Hankey GJ, editors. Stroke prevention and treatment: an evidence-based approach. 2nd ed. Cambridge: Cambridge University Press; 2020. p. 10-34.

42 Schulz KF, Altman DG, Moher D; CONSORT Group. CONSORT 2010 statement: updated guidelines for reporting parallel group randomised trials. BMJ. 2010;340:c332.

43 Turner L, Shamseer L, Altman DG, Weeks L, Peters J, Kober T, et al. Consolidated standards of reporting trials (CONSORT) and the completeness of reporting of randomised controlled trials (RCTs) published in medical journals. Cochrane Database Syst Rev. 2012; 11(11):MR000030.

44 Peto R. Why do we need systematic overviews of randomized trials? Stat Med. 1987;6:23344.

45 Collins R, Gray R, Godwin J, Peto R. Avoidance of large biases and large random errors in the assessment of moderate treatment effects: the need for systematic overviews. Stat Med. 1987;6:245-54.

46 Lewis S, Clarke M. Forest plots: trying to see the wood and the trees. BMJ. 2001;322:1479_ 80.

47 Gardner MJ, Altman DG. Estimating with confidence. Br Med J (Clin Res Ed). 1988;296: $1210-1$.

48 Sandercock PA. Short history of confidence intervals: or, don't ask "does the treatment work?" but "how sure are you that it works? Stroke. 2015;46:e184-7.
49 Rothwell PM. Treating individuals 1. External validity of randomised controlled trials: "to whom do the results of this trial apply? Lancet. 2005;365:82-93.

50 Rothwell PM. Treating individuals 2. Subgroup analysis in randomised controlled trials: importance, indications, and interpretation. Lancet. 2005;365:176-86.

51 Guyatt G, Oxman AD, Akl EA, Kunz R, Vist $\mathrm{G}$, Brozek J, et al. GRADE guidelines: 1 . Introduction-GRADE evidence profiles and summary of findings tables. J Clin Epidemiol. 2011;64:383-94.

52 Langhorne P, Williams BO, Gilchrist W, Howie K. Do stroke units save lives? Lancet. 1993;342:395-8.

53 Stroke Unit Trialists' Collaboration. Collaborative systematic review of the randomised trials of organised inpatient (stroke unit) care after stroke. BMJ. 1997;314:1151-9.

54 Vahedi K, Hofmeijer J, Juettler E, Vicaut E, George B, Algra A, et al. Early decompressive surgery in malignant infarction of the middle cerebral artery: a pooled analysis of three randomised controlled trials. Lancet Neurol. 2007;6:215-22.

55 Hankey GJ. Stroke. Lancet. 2017;389:641-54.

56 Campbell BCV, Khatri P. Stroke. Lancet. 2020;396:129-42.

57 National Institute of Neurological Disorders and Stroke rt-PA Stroke Study Group. Tissue plasminogen activator for acute ischemic stroke. N Engl J Med. 1995;333:1581-7.

58 Wardlaw JM, Murray V, Berge E, del Zoppo GJ. Thrombolysis for acute ischaemic stroke. Cochrane Database Syst Rev. 2014;2014(7): CD000213.

59 Rodrigues FB, Neves JB, Caldeira D, Ferro JM, Ferreira JJ, Costa J. Endovascular treatment versus medical care alone for ischaemic stroke: systematic review and meta-analysis. BMJ. 2016;353:i1754.

60 Goyal M, Menon BK, van Zwam WH, Dippel DW, Mitchell PJ, Demchuk AM, et al. Endovascular thrombectomy after large-vessel ischaemic stroke: a meta-analysis of individual patient data from five randomised trials. Lancet. 2016;387:1723-31.

61 Mir H, Siemieniuk RAC, Ge L, Foroutan F, Fralick M, Syed T, et al. Patent foramen ovale closure, antiplatelet therapy or anticoagulation in patients with patent foramen ovale and cryptogenic stroke: a systematic review and network meta-analysis incorporating complementary external evidence. BMJ Open. 2018;8:e023761.

62 Trifan G, Gorelick PB, Testai FD. Efficacy and safety of using dual versus monotherapy antiplatelet agents in secondary stroke prevention: systematic review and meta-analysis of randomized controlled clinical trials. Circulation. 2021 Apr 30.

63 Page MJ, Sterne JAC, Higgins JPT, Egger M. Investigating and dealing with publication bias and other reporting biases in meta-analyses of health research: a review. Res Synth Methods. 2021;12:248-59. 
64 Sterne JAC, Savović J, Page MJ, Elbers RG, Blencowe NS, Boutron I, et al. RoB 2: a revised tool for assessing risk of bias in randomised trials. BMJ. 2019;366:14898.

65 Page MJ, McKenzie JE, Kirkham J, Dwan K, Kramer S, Green S, et al. Bias due to selective inclusion and reporting of outcomes and analyses in systematic reviews of randomised trials of healthcare interventions. Cochrane Database Syst Rev. 2014;10:MR000035.

66 Sterne JA, Sutton AJ, Ioannidis JP, Terrin N, Jones DR, Lau J, et al. Recommendations for examining and interpreting funnel plot asymmetry in meta-analyses of randomised controlled trials. BMJ. 2011;343:d4002.

67 Savovic J, Turner RM, Mawdsley D, Jones HE, Beynon R, Higgins JPT, et al. Association between risk-of-bias assessments and results of randomized trials in cochrane reviews: the ROBES Meta-Epidemiologic Study. Am J Epidemiol. 2018;187:1113-22.

68 Page MJ, McKenzie JE, Bossuyt PM, Boutron I, Hoffmann TC, Mulrow CD, et al. The PRISMA 2020 statement: an updated guideline for reporting systematic reviews. BMJ. 2021;372: n71.

69 Chalmers I, Dickersin K, Chalmers TC. Getting to grips with Archie Cochrane's agenda. BMJ. 1992;305:786-8.

70 Chalmers I, Haynes B. Reporting, updating, and correcting systematic reviews of the effects of health care. BMJ. 1994;309:862-5.

71 Berge E, Langhorne P. Cochrane Stroke Group: twenty years of evidence-based stroke medicine. Int J Stroke. 2014;9:107-9.

72 CAPRIE Steering Committee. A randomised, blinded, trial of clopidogrel versus aspirin in patients at risk of ischaemic events (CAPRIE). CAPRIE Steering Committee. Lancet. 1996; 348:1329-39.

73 Yusuf S, Zhao F, Mehta SR, Chrolavicius S, Tognoni G, Fox KK, et al. Effects of clopidogrel in addition to aspirin in patients with acute coronary syndromes without ST-segment elevation. N Engl J Med. 2001;345:494-502.
74 CAST (Chinese Acute Stroke Trial) Collaborative Group. CAST: randomised placebocontrolled trial of early aspirin use in 20,000 patients with acute ischaemic stroke. Lancet. 1997;349:1641-9.

75 The IST-3 Collaborative Group. The benefits and harms of intravenous thrombolysis with recombinant tissue plasminogen activator within $6 \mathrm{~h}$ of acute ischaemic stroke (the third international stroke trial [IST-3]): a randomised controlled trial. Lancet. 2012;379: 2352-63.

76 PROGRESS Collaborative Group. Randomised trial of a perindopril-based bloodpressure-lowering regimen among 6,105 individuals with previous stroke or transient ischaemic attack. Lancet. 2001;358:1033-41.

77 Heart Protection Study Collaborative Group. MRC/BHF Heart Protection Study of cholesterol lowering with simvastatin in 20,536 high-risk individuals: a randomised placebocontrolled trial. Lancet. 2002;360:7-22.

78 Amarenco P, Bogousslavsky J, Callahan A III, Goldstein LB, Hennerici M, Rudolph AE, et al. High-dose atorvastatin after stroke or transient ischemic attack. N Engl J Med. 2006;355: 549-59.

79 Rothwell PM. Clinical innovation in stroke: getting the simple things right. Lancet Neurol. 2018;17:491-3.

80 O'Collins VE, Macleod MR, Donnan GA, Horky LL, van der Worp BH, Howells DW. 1,026 experimental treatments in acute stroke. Ann Neurol. 2006;59(3):467-77.

81 Bath PM, Appleton JP, England T. The hazard of negative (not neutral) trials on treatment of acute stroke: a review. JAMA Neurol. 2020; 77:114-24.

82 Stroke Therapy Academic Industry Roundtable (STAIR). Recommendations for standards regarding preclinical neuroprotective and restorative drug development. Stroke. 1999;30:2752-8.
83 Fisher M; Stroke Therapy Academic Industry Roundtable. Recommendations for advancing development of acute stroke therapies: Stroke Therapy Academic Industry Roundtable 3. Stroke. 2003;34:1539-46.

84 Gibson LM, Brazzelli M, Thomas BM, Sandercock PA. A systematic review of clinical trials of pharmacological interventions for acute ischaemic stroke (1955-2008) that were completed, but not published in full. Trials. 2010; 11:43.

85 Berge E, Ford GA, Bath PM, Stapf C, van der Worp HB, Demotes J, et al. Regulation and governance of multinational drug trials in stroke: barriers and possibilities. Int J Stroke. 2015; 10:425-8

86 Al-Shahi R, Vousden C, Warlow C; Scottish Intracranial Vascular Malformation Study (SIVMS) Steering Committee. Bias from requiring explicit consent from all participants in observational research: prospective, population-based study. BMJ. 2005;331:942-5.

87 Reith C, Landray M, Devereaux PJ, Bosch J, Granger CB, Baigent C, et al. Randomized clinical trials: removing unnecessary obstacles. N Engl J Med. 2013;369:1061-5.

88 Berge E, Al-Shahi Salman R, van der Worp HB, Stapf C, Sandercock P, Sprigg N, et al. Increasing value and reducing waste in stroke research. Lancet Neurol. 2017;16:399-408.

89 Meeker-O'Connell A, Glessner C, Behm M, Mulinde J, Roach N, Sweeney F, et al. Enhancing clinical evidence by proactively building quality into clinical trials. Clin Trials. 2016;13: 439-44.

90 Meeker-O'Connell A, Glessner C. Clinical trial quality: from supervision to collaboration and beyond. Clin Trials. 2018;15:23-6.

91 Baigent C. Pragmatic trials: need for ADAPTABLE design. N Engl J Med. 2021 May 15.

92 Hankey GJ. Isolated angiitis/angiopathy of the central nervous system. Cerebrovascular Diseases. 1991;1:2-15. 